\title{
Strength degradation and lifetime prediction of dental zirconia ceramics under cyclic normal loading
}

\author{
Wanzhong $\mathrm{Li}^{\mathrm{a},{ }^{*}}$, Yingqiang $\mathrm{Xu}^{\mathrm{a}}$, Huiming $\mathrm{He}^{\mathrm{b}}$, Haidan $\mathrm{Zhao}^{\mathrm{c}}$, Jian $\mathrm{Sun}^{\mathrm{a}}$ and Yue Hou ${ }^{\mathrm{a}}$ \\ ${ }^{a}$ School of Mechanical Engineering, Northwestern Polytechnical University, Xi'an, Shaanxi, P.R. \\ China \\ ${ }^{\mathrm{b}}$ School of Stomatology, The Fourth Military Medical University, Xi'an, Shaanxi, P.R. China \\ ${ }^{c}$ The $309^{\text {th }}$ Hospital of Chinese People's Liberation Army, Beijing, P.R. China
}

\begin{abstract}
Clinical cases show that zirconia restoration could happen fracture by accident under overloading after using a period of time. The purpose of this study is to research mechanical behavior and predict lifetime of dental zirconia ceramics under cyclic normal contact loading with experiments. Cyclic normal contact loading test and three point bending test are carried on specimens made of two brands of dental zirconia ceramic to obtain flexure strength and damage degree after different number of loading cycles. By means of damage mechanics model, damage degree under different number of contact loading cycles are calculated according to flexure strength, and verified by SEM photographs of cross section morphology of zirconia ceramics specimen phenomenologically. Relation curve of damage degree and number of cycles is fitted by polynomial fitting, then the number of loading cycles can be concluded when the specimen is complete damage. Strength degradation of two brands dental zirconia ceramics are researched in vitro, and prediction method of contact fatigue lifetime is established.
\end{abstract}

Keywords: Strength degradation, lifetime prediction, damage degree, flexure strength, normal cyclic load

\section{Introduction}

Zirconia ceramic has excellent transmittance, biocompatibility, anticorrosion, anti-abrasive and mechanical properties, firstly applied in industrial field [1-4]. In 1960s, Chrisel introduced zirconia as biological materials used in orthopaedic joint replacement [5]. Half a century, the whole world had more than six hundred thousand patients implanted with zirconia joints, zirconia ceramic had been widely used in medical field as a kind of biological materials. With the development of CAD/CAM technology and improvement of zirconia ceramics performance, zirconia ceramic could be used to repair tetracycline pigmentation teeth, dental fluorosis, tooth deformity, discolored tooth and so on, which could meet the needs of different patients better [6-11]. Moreover, the function and color of zirconia ceramic dentures is approximate to dermal tooth. Thus, dental zirconia ceramics have become

*Address for correspondence: Wanzhong Li, PO Box 324, 127 West Youyi Road, Xi'an Shaanxi, 710072, P.R. China. Tel.: +86 15691821236; Fax: +86 29 88493928; E-mail: imposing@yeah.net. 
the most important material of artificial teeth.

However, as a kind of brittle material, zirconia restoration suffered from erosion of damp environment and repeating masticatory force in the mouth, may result in unexpected fracture failure [12-16]. Zirconia ceramics showed higher clinical success rate than the other ceramics: success rate could be above $95 \%$ in $2 \sim 3$ years, as for $3 \sim 5$ years, it also could achieve more than $90 \%$ [17-20]. Under the effect of masticatory force for a long time, the strength of zirconia ceramics would reduce [21]. Clinical failure usually is owing to fracture of veneering ceramic, but base crown of zirconia restoration still could happen fracture in 3 5 years of clinical research. Moreover, clinical cases showd that zirconia restoration could happen fracture by accident under overloading after using a period of time. For half a century, fracture failure and subcritical crack growth (SCG) were researched in the ceramics literature, but research of relationship between strength degradation and fracture failure still almost no one involved [22-24]. Hence, it is worth exploring for strength degradation and lifetime prediction of dental zirconia ceramics under cyclic normal loading.

This work adopted cyclic contact fatigue loading test and three point bending test to explore and establish a rapid and high-efficiency method of contact fatigue lifetime prediction for dental zirconia ceramics in vitro. According to flexure strength under different number of contact loading cycles, corresponding damage degree $D$ were calculated by means of damage mechanics model, and verified by SEM photographs of cross section morphology of zirconia ceramics specimen phenomenologically. Relation curve of damage degree $D$ and number of cycles was fitted by polynomial fitting, then the number of loading cycles could be concluded when the specimen was damaged completely.

\section{Materials and methods}

\subsection{Experiment principle overview}

Contact between spherical and flat surface based on Hertz theory is regarded as one of the most effective method for the contact fatigue evaluation of dental ceramics [25-28]. As shown in Figure 1, because the hardness of tungsten carbide is greater than that of zirconia ceramics, the contact of this test is designed between tungsten carbide ball and zirconia ceramics flat surface specimen, and radius of tungsten carbide ball is same with the equivalent curvature radius of dental cusp. Masticatory force is simulated by normal contact load $F$, it is applied on specimen surface of zirconia ceramics in order to research its contact fatigue behavior of dental zirconia ceramics.

Under cyclic normal contact load $F$, the contact radius:

$$
a=\left(\frac{3 F R}{4 E^{*}}\right)^{1 / 3}
$$

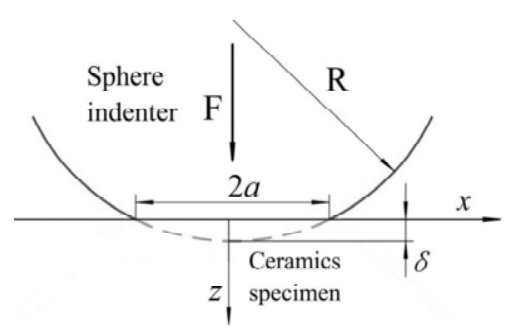

Fig. 1. Hertz contact between tungsten carbide ball and zirconia ceramics flat surface specimen. 


$$
E^{*}=\left(\frac{1-v_{1}^{2}}{E_{1}}+\frac{1-v_{2}^{2}}{E_{2}}\right)^{-1}
$$

Where $F$ is normal contact load, $R$ is radius of tungsten carbide ball, $E^{*}$ is equivalent elastic modulus of two contact materials, $E_{1}, E_{2}, v_{1}$ and $v_{2}$ are elastic modulus and Poisson's ratio of tungsten carbide ball and zirconia ceramics flat surface specimen, respectively. It is generally recognized that the ring cracks are caused by tensile stress [29]. The maximum tensile stress $\sigma_{\mathrm{m}}$ in the specimen occurs at the contact circle $(r=a, z=0)$ :

$$
\sigma_{m}=\frac{\left(1-2 v_{2}\right)}{3} p_{0}
$$

Where $p_{0}$ is the maximum contact pressure, and $p_{0}=3 F /\left(2 \pi a^{2}\right)$. In terms of damage mechanics, the extent of material damage is described by damage degree $D$, and $0 \leq D \leq 1$. In the condition of nondestructive, $D=0 ; D=1$ for the complete damage. The strain equivalence hypothesis proposed by Lemaitre [30] is extensively used to establish the damage constitutive equation. This hypothesis can be described as follows: for the damaged elastic brittle material under effect of true stress $\sigma$, the strain of damaged state could be equivalent to the strain of virtual element state under effect of effective stress $\widetilde{\sigma}$. Based on this hypothesis, the stress-strain relation of elastic brittle damage material can be expressed as

$$
\begin{gathered}
\varepsilon=\frac{\widetilde{\sigma}}{E}=\frac{\sigma}{E(1-D)} \\
D=1-\frac{\sigma}{\widetilde{\sigma}}
\end{gathered}
$$

Three-point bending test is designed to get material flexure strength and also damage degree $D$ under different number of loading cycles. At first, strain $\varepsilon$ is calculated by means of putting elasticity modulus $E$ and flexure strength of nondestructive material in Eq. (4). Then according to strain equivalence hypothesis, regard this strain $\varepsilon$ as a fixed value, and flexure strength under different number of cyclic loading is substituted into Eqs. (4) and (5), the damage degree of specimens under different number of cyclic loading is calculated. At this point, relationship between damage degree and flexure strength can be obtained. Moreover, relation curve of damage degree $D$ and number of cycles is fitted by polynomial fitting, the number of cycles can be obtained when $D=1$.

The maximum tensile stress theory indicates that maximum tensile stress is the main factor causing fracture for brittle materials. In order to ensure that the tensile stress zone under cyclic contact loading is still suffered from tensile stress under three-point bending test, it is noteworthy that though the loading position is specimen center, the loading surface of cyclic normal contact loading test must be the opposite surface of three point bending test.

\subsection{Cyclic normal contact loading test}

This test aims to cause different damage degree of zirconia ceramics specimens through different 
Table 1

Specifications and mechanical parameters of relevant materials

\begin{tabular}{llllll}
\hline Material & $\begin{array}{l}\text { Size } \\
\mathrm{mm}\end{array}$ & $\begin{array}{l}\text { Elastic modulus } \\
\mathrm{E} / \mathrm{GPa}\end{array}$ & $\begin{array}{l}\text { Poisson's ratio } \\
v\end{array}$ & $\begin{array}{l}\text { Yield Strength } \\
\mathrm{Sy} / \mathrm{GPa}\end{array}$ & $\begin{array}{l}\text { Roughness } \\
\mathrm{Ra} / \mu \mathrm{m}\end{array}$ \\
\hline tungsten carbide ball & $\varnothing 4$ & 600 & 0.25 & 3.9 & 0.01 \\
tungsten carbide support roller & $\varnothing 4 \times 6$ & 600 & 0.25 & 3.9 & 0.08 \\
WL zirconia specimen & $20 \times 4 \times 2$ & 210 & 0.27 & 2.0 & 0.08 \\
AT zirconia specimen & $20 \times 4 \times 2$ & 210 & 0.27 & 2.0 & 0.08 \\
\hline
\end{tabular}

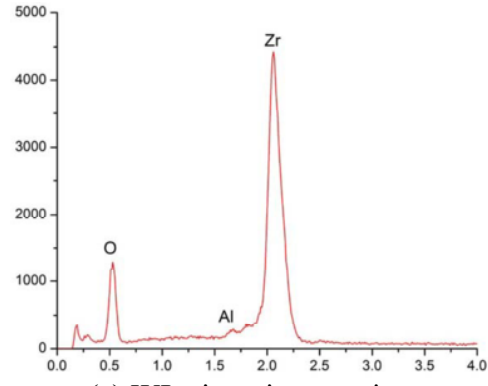

(a) WL zirconia ceramics

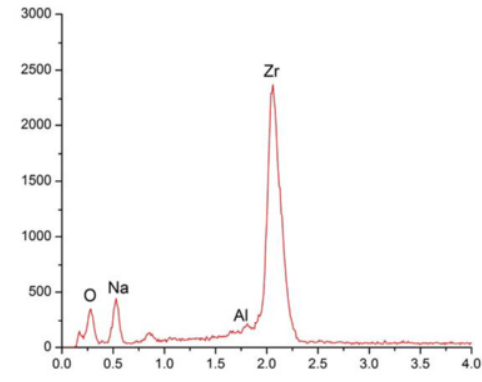

(b) AT zirconia ceramics

Fig. 2. Energy spectrum of WL and AT zirconia ceramics.

number of cyclic contact loading. To simulate the actual working situation of dental materials in the mouth, two brands of zirconia ceramics (WL and AT) are made into 20 specimens of same size respectively, and divided into four groups, each group of five. Specifications and mechanical parameters of tungsten carbide ball and zirconia ceramics flat surface specimen are shown in Table 1, and two kinds of zirconia ceramics energy spectrum are shown in Figure 2. Although WL and AT are both zirconia ceramic material, their compositions are different slightly because of the different manufacturers.

Cyclic loading experiment device is designed as Figure 3, the zirconia ceramics flat surface specimen and tungsten carbide ball are fixed on loading platform of Instron 5848 electromechanical test machine with cyanoacrylate adhesive, and the center of specimen is pressed by tungsten carbide ball. Afterwards, load mode is set as sinusoidal normal stress, and load frequency is $2 \mathrm{~Hz}$. According to actual masticatory force, cyclic loading force is set to $50 \sim 500 \mathrm{~N}$. And the numbers of cycles are 0 , $10^{3}, 10^{4}$ and $10^{5}$ of each group. According to the relevant experimental content of $[23,27,28,31]$, considering the room temperature and oral temperature are similar, this temperature difference have very little impact on dental zirconia ceramics, and in the process of chewing, food type, liquid ingredients, accidental stress and other factors have too much uncertainty. Thus, the whole test are proceeded in vitro with environment temperature of $25^{\circ} \mathrm{C}$ and relative humidity of $40 \%$.

\subsection{Three-point bending test}

After Cyclic contact fatigue loading test, specimen is washed by acetone to separate from the loading platform. In accordance with the requirements of ISO6872-2008, three point bending test of specimens is implemented by AGS-10kN universal testing machine. The significance of three point bending test is to obtain flexural strength of two brands of zirconia ceramics. Figure 4 shows loading position and size requirements of three point bending test. Loading position is the center of specimen, diameter of support rollers is $4 \mathrm{~mm}$ and support span $\mathrm{L}$ is $16 \mathrm{~mm}$. Loading rate is set to $0.5 \mathrm{~mm} / \mathrm{min}$, 


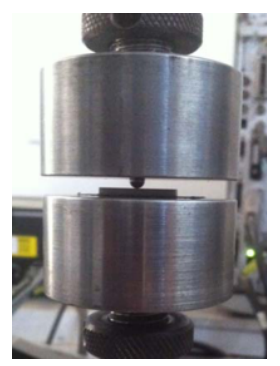

Fig. 3. Cyclic contact test.

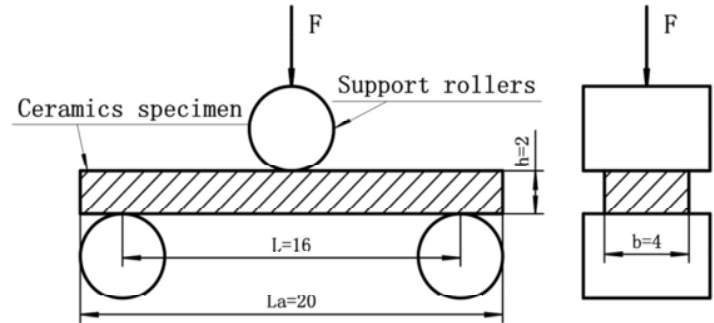

Fig. 4. Schematic diagram of three point bending test.

until the specimen fractured. Flexure strength is calculated using the following equation:

$$
\sigma_{f}=\frac{3 F L}{2 b h^{2}}
$$

Where $\sigma_{f}$ is flexure strength, $F$ is the fracture load, $b$ and $h$ is the width and thickness of the specimen, respectively.

\section{Results}

When loading rate is $0.5 \mathrm{~mm} / \mathrm{min}$, fracture loads of zirconia ceramics specimens suffering different number of loading cycles are recorded until the specimen broken.

Flexure strength is calculated from Eq. (6). Figure 5 shows the fracture load and strength results of three point bending test under different number of loading cycles. Due to the subtle differences of surface roughness, geometry size among different specimens, the test results have certain discrete degree. It is observed that flexure strength of two kinds of zirconia ceramics are on the decline with the increasing number of cycles. For zirconia ceramics of WL, the flexure strength of each cyclic loading group has no significant difference. By contrast, zirconia ceramics of AT has obvious strength degradation. Especially after $10^{5}$ times of cyclic loading, mean flexure strength of AT fall to 499.28 $\mathrm{MPa}$ from $622.76 \mathrm{MPa}$, the rate of strength descend is $19.83 \%$. By comparing the flexure strength of same number of cycles, it is found that flexure strength of WL is superior to AT. This shows WL ceramic has better mechanical performance and fatigue resistance performance in the conditions of this test.

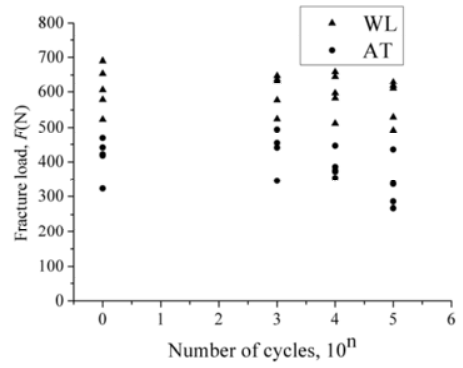

(a) Fracture load

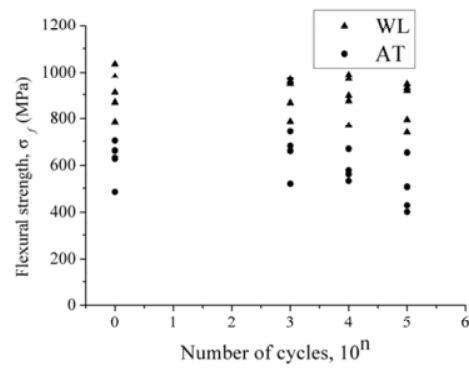

(b) Flexure strength

Fig. 5. Fracture load and strength results of three point bending test under different number of loading cycles. 
Weibull distribution analysis is often used to describe the fracture probability of material, and research strength degradation from the point of statistics [32]. Weibull analytic function can be expressed as [16, 33]:

$$
P_{f}=1-\exp \left[-\left(\sigma_{f} / \sigma_{0}\right)^{m}\right]
$$

Where $P_{f}$ is failure probability, $\sigma_{f}$ is flexure strength, $\sigma_{0}$ is characteristic strength for $P_{f}=63.21 \%, m$ is Weibull modulus. In this work, Weibull distribution is used to analyze the flexure strength data of WL and AT under different number of cycles from three-point bending test. For WL, Weibull modulus is $9.99,12.73,11.77,7.88$ under loading cycles of $0,10^{3}, 10^{4}$ and $10^{5}$ respectively; and for AT, Weibull modulus is $6.61,7.85,11.77,5.88$ under loading cycles of $0,10^{3}, 10^{4}$ and $10^{5}$ respectively. It is observed that Weibull modulus shows a trend of descending after ascending. Moreover, Weibull modulus is measurement factor of material damage dispersion degree. The smaller the Weibull modulus, the experimental results of flexure strength are more discrete, and fracture could be happen easier.

Figure 6 shows scanning electron microscope (SEM) photographs of cross section morphology of two kinds of dental zirconia ceramics under different number of loading cycles. It is observed that the damage of fracture section is more and more obvious with the increasing number of cycles for the same brand of zirconia ceramics. Further, for the different brands of zirconia ceramics, damage of fracture section have obvious difference under same number of cycles.

\section{Discussion}

Flexure strength of material is closely associated with its mechanical properties, it is influenced by material thickness, radius of loading ball, magnitude of load and so on. To eliminate the influence of geometrical shape and loading magnitude, loading test adopts same specimen size, same radius of
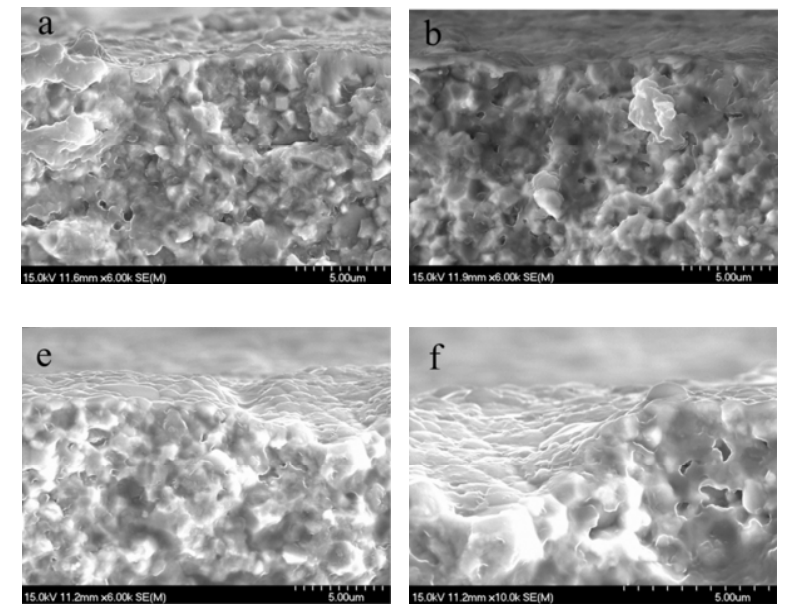
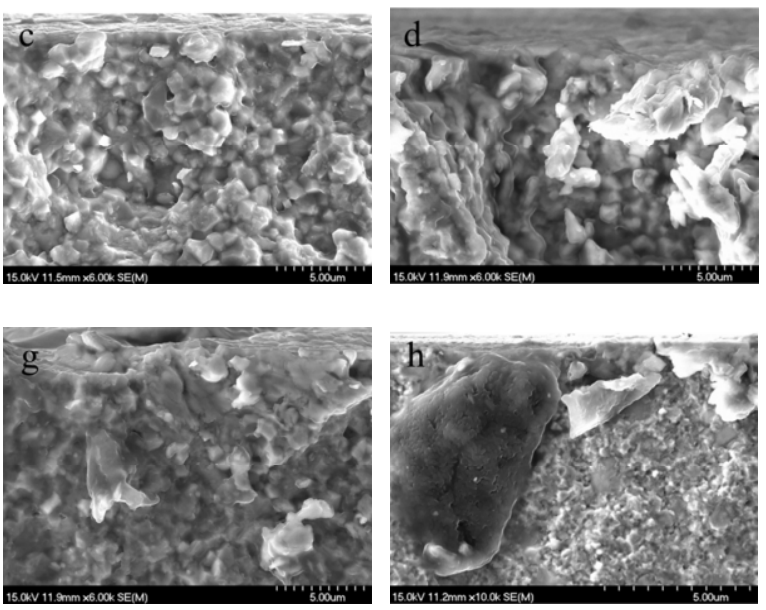

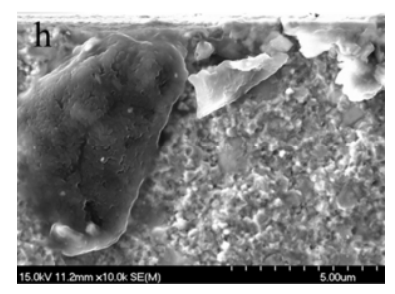

Fig. 6. SEM photographs of cross section morphology of two kinds of dental zirconia ceramics under different number of loading cycles. (a) WL, 0 times cycle, $D=0$; (b) WL, $10^{3}$ times cycle, $D=0.127$; (c) WL, $10^{4}$ times cycle, $D=0.142$; (d) WL, $10^{5}$ times cycle, $D=0.186$; (e) AT, 0 times cycle, $D=0$; (f) AT, $10^{3}$ times cycle, $D=0.156$; (g) AT, $10^{4}$ times cycle, $D=0.291$; (h) AT, $10^{5}$ times cycle, $D=0.328$. 
loading ball, and same loading type, so the test results are real and objective. Nonetheless, the test results of flexure strength have certain discrete degree, which is because compositions, internal defect and surface roughness are different slightly.

The variation of Weibull modulus with trend of increasing first and then decreasing may be caused by phase transition of zirconia ceramics under cyclic normal contact loading. Commonly, microscopic structure of sintered dental zirconia ceramics after has most of tetragonal phase and a small amount of monoclinic phase. Micro cracks are generated by cyclic loading, and tetragonal phase around crack tips is transformed into monoclinic phase by tensile stress. Further crack propagation is prevented by volume expansion and shear strain from phase transition, volume expansion counteract strength degradation caused by micro cracks. After that, with the increase of cycling times, increasing damage degree and strength degradation are caused by further crack propagation. Hence, the trend of Weibull modulus is going up at the beginning, and then down.

According to Eq. (5) and flexure strength results of Figure 5, the damage degree $D$ is calculated. Qualitatively, the calculation results are consistent with the SEM photographs. At this point, it can be inferred that flexure strength differences of two brands zirconia ceramics mainly relate to the material composition and processing method. Figure 7 provides relationship between damage degree and flexural strength, and it is observed that the more severe damage degree, the strength degradation is greater. In addition, when damage degree is in the same, the flexural strength of WL is greater than that of AT. Figure 8 shows damage degree $D$ and their cubic polynomial fitting curves of WL and AT zirconia ceramics under different number of loading cycles.

Theoretically, material reaches to the complete damage when damage degree $D$ equals to one. In the condition of $50 \sim 500 \mathrm{~N}$ cyclic loading of this experiment, the corresponding stress amplitude is 360.9 $\mathrm{MPa}$. The predicted lifetimes of two kinds of zirconia ceramics are $8.56 \times 10^{8}$ and $6.26 \times 10^{7}$ loading cycles respectively. WL ceramic has better mechanical performance and fatigue resistance performance than AT ceramic. Particularly worth mentioning is the average chewing number is $4 \times 10^{3}$ times one people a day, adds up to $1 \times 10^{6}$ times a year [34]. Hence, conservatively speaking, WL and AT zirconia ceramics could service in the mouth more than twenty years under normal masticatory force without overloading. Bicalho, et al. [35] researched fatigue lifetime of zirconia ceramics using four-point bending test, they concluded that the cycle times of zirconia ceramics was 255270 under cyclic stress of $285 \mathrm{MPa}$ and lifetime exceeded twenty years in the condition of masticatory force. This conclusion is consistent with that of this work.

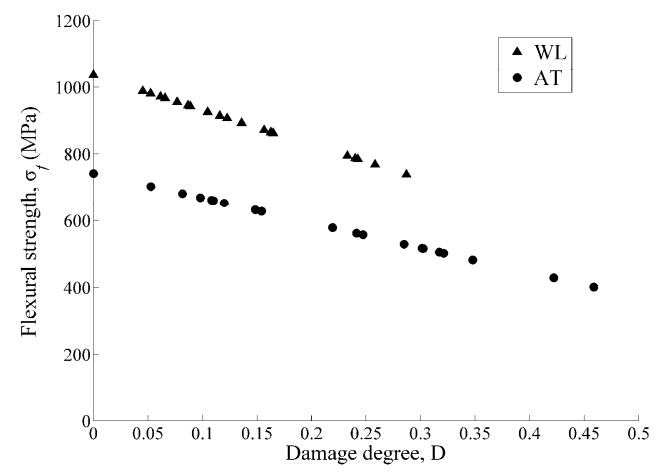

Fig. 7. Relation curve of damage degree and flexural strength.

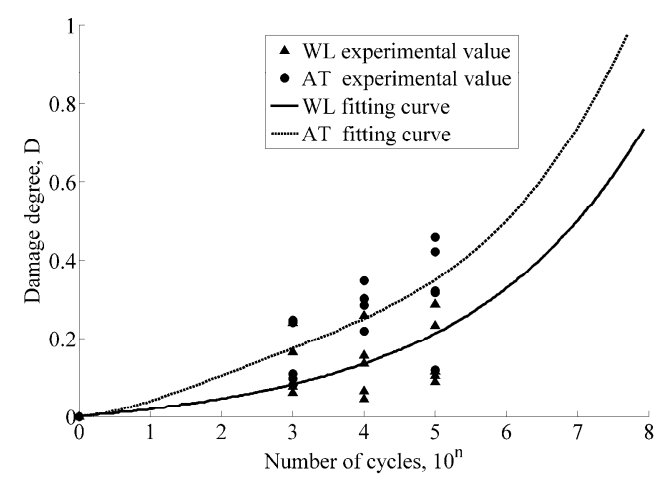

Fig. 8. Relation curve of number of cycles and damage degree. 


\section{Conclusions}

The service lifetime of dental ceramics has been attracting the attention of dentists and patients, and the strength degradation and lifetime prediction of ceramic also needs to be further researched urgently. On the basis of Hertz contact theory and damage mechanics, combining with cyclic normal contact loading test and three point bending test, mechanical behaviors of two kinds of different brands dental zirconia ceramics were researched in vitro. It was conducive to design and restore dental prosthesis for dentists.

The results showed that contact fatigue lifetime prediction results of two kinds of dental zirconia ceramics were conformity with clinical reality. As the number of cycles increases, damage degree of zirconia ceramics was increasing, but flexure strength was on the decline. In contrast, strength of WL exceeded that of AT, and zirconia ceramics of WL had longer service lifetime. The effects of processing method, material composition, service condition and microstructure to strength and lifetime of zirconia ceramics, need to be further researched.

\section{Acknowledgments}

This work is supported by the Doctorate Foundation of Northwestern Polytechnical University (CX201420).

\section{References}

[1] T. Vagkopoulou, S.O. Koutayas, P. Koidis and J.R. Strub, Zirconia in dentistry: Part 1, discovering the nature of an upcoming bioceramic, The European Journal of Esthetic Dentistry 4 (2009), 130-151.

[2] A.S. Kassem, O. Atta and O. EI-Mowafy, Fatigue resistance and microleakage of CAD/CAM ceramic and composite molar crowns, Journal of Prosthodontics 21 (2012), 28-32.

[3] F.J.T. Burke, R.J. Crisp, A.J. Cowan, et al., Five-year clinical evaluation of zirconia-based bridges in patients in UK general dental practices, Journal of Dentistry 41 (2013), 992-999.

[4] D. Lops, E. Bressan, M. Chiapasco, et al., Zirconia and titanium implant abutments for single-tooth implant prostheses after 5 years of function in posterior regions, The International Journal of Oral \& Maxillofacial Implants 28 (2013), 281-287.

[5] J. Helmer and T. Driskell, Research on bioceramics, Proceedings of the Symposium on Use of Ceramics as Surgical Implants, South Carolina, 1969.

[6] L.J. Shang, Y. Wu and Y.J. Xu, Effect of the CAD/CAM zirconia all-ceramic crown restoration on periodontal tissue, Chinese Journal of Tissue Engineering Research 30 (2014), 4804-4809.

[7] T.F. Zhou and X.Z. Wang, Finite element analysis of tooth restored with one-piece of computer aided design and computer aided manufacturing zirconia post-core in three different radiuses, Journal of Peking University (Health Sciences) 44 (2012), 93-97.

[8] T.F. Zhou, X.H. Zhang and X.Z. Wang, Three-dimensional finite element analysis of one-piece computer aided design and computer aided manufacture involved zirconia post and core, Journal of Peking University (Health Sciences) 47 (2015), 78-84.

[9] M. Tischler, S.D. Ganz and C. Patch, An ideal full-arch tooth replacement option: CAD/CAM zirconia screw-retained implant bridge, Dentistry Today 32 (2013), 98-102.

[10] P. Liu, X.L. Deng and X.Z. Wang, Use of a CAD/CAM-fabricated glass fiber post and core to restore fractured anterior teeth: a clinical report, Journal of Prosthetic Dentistry 103 (2010), 330-333.

[11] Z.Y. Chen, Y. Li, X.L. Deng, et al, A novel computer-aided method to fabricate a custom one-piece glass fiber doweland- core based on digitized impression and crown preparation data, Journal of Prosthodontics 23 (2014), 276-283.

[12] P.F. Manicone, P.R. Rossi Iommetti and L. Raffaelli, An overview of zirconia ceramics: Basic properties and clinical applications, Journal of Dentistry 35 (2007), 819-826. 
[13] Y. Zhang, Overview: Damage resistance of graded ceramic restorative materials, Journal of the European Ceramic Society 32 (2012), 2623-2632.

[14] P. Triwatana, N. Nagaviroj and C. Tulapornchai, Clinical performance and failures of zirconia-based fixed partial dentures: a review literature, Journal of Advanced Prosthodontics 4 (2012), 76-83.

[15] M.N. Aboushelib, Fatigue and fracture resistance of zirconia crowns prepared with different finish line designs, Journal of Prosthodontics 21 (2012), 22-27.

[16] H.D. Zhao, Y.P. Hu, C.B. Zhang, et al., Fatigue behavior of zirconia ceramics after cyclic loading in vitro, Chinese Journal of Conservative Dentistry 23 (2013), 344-348.

[17] F. Beuer, D. Edelhoff, W. Gernet and J.A. Sorensen, Three-year clinical prospective evaluation of zirconia-based posterior fixed dental prostheses (FDPs), Clinical Oral Investigations 13 (2009), 445-451.

[18] I. Sailer, A. Feher and F. Filser, et al., Five-year clinical results of zirconia frameworks for posterior fixed partial dentures, International Journal of Prosthodontics 20 (2007), 383-388.

[19] B. Taskonak and A. Sertgöz, Two-year clinical evaluation of Lithia-disilicate-based all-ceramic crowns and fixed partial dentures, Dental Materials 22 (2006), 1008-1013.

[20] H.J. Conrad, W.J. Seong and G.J. Pesun, Current ceramic materials and systems with clinical recommendations: A systematic review, Journal of Prosthetic Dentistry 98 (2007), 389-404.

[21] A.R. Studart, F. Filser, P. Kocher, et al., Cyclic fatigue in water of veneer-framework composites for all-ceramic dental bridges, Dental Materials 23 (2007), 177-185.

[22] A.G. Evans, Fatigue in ceramics, International Journal of Fracture 16 (1980), 485-498.

[23] N.A. Fleck, K.J. Kang and M.F. Ashby, Overview no. 112: The cyclic properties of engineering materials, Acta Metallurgica et Materialia 42 (1994), 365-381.

[24] G. Roebben, M. Steen, J. Bressers, et al., Mechanical fatigue in monolithic non-transforming ceramics, Progress in Materials Science 40 (1996), 265-331.

[25] K.Y. Lee and K.C. Ludema, Fatigue wear mechanism of alumina, simulated by synchronized biaxial repeated indentations, Wear 205 (1997), 200-205.

[26] T. Fett, E. Ernst, G. Rizzi, et al, Sphere contact fatigue of a coarse-grained $\mathrm{Al}_{2} \mathrm{O}_{3}$ ceramic, Fatigue \& Fracture of Engineering Materials \& Structures 29 (2006), 876-886.

[27] B.R. Lawn, Indentation of ceramics with spheres: A century after Hertz, Journal of the American Ceramic Society 81 (1998), 1977-1994.

[28] Y. Wang and B.W. Darvell, Failure mode of dental restorative materials under Hertzian indentation, Dental Materials 23 (2007), 1236-1244.

[29] L. Ceseracciu, M. Anglada and E. Jiménez-Piqué, Hertzian cone crack propagation on polycrystalline materials: Role of R-curve and residual stresses, Acta Materialia 56 (2008), 265-273.

[30] J. Lemaitre and J.L. Chaboche, Mechanics of Solid Materials, Cambridge University Press, Cambridge, 1994.

[31] X.D. Dong and B.W. Darvell, Stress distribution and failure mode of dental ceramic structures under Hertzian indentation, Dental Materials 19 (2003), 542-551.

[32] S. Nohut and C. Lu, Fracture statistics of dental ceramics: Discrimination of strength distributions, Ceramics International 38 (2012), 4979-4990.

[33] W. Weibull, A statistical distribution function of wide applicability, Journal of Applied Mechanics-Transactions of the ASME 18 (1951), 293-297.

[34] W. Zhou, L. Zhang, P. Li and S.F. Zhang, The analytic research on random parameters in the fatigue strength reliability analysis of all-ceramic crown, Journal of Clinical Stomatology 26 (2010), 457-459.

[35] L.A. Bicalho, C.A.R.P. Bapthsta, R.C. Souza, et al., Fatigue and subcritical crack growth in $\mathrm{ZrO}_{2}$-bioglass ceramics, Ceramics International 39 (2013), 2405-2414. 